\title{
A Neutrosophic Multicriteria Decision Making Method
}

\author{
ATHAR KHARAL ${ }^{* \dagger}$ \\ National University of Sciences and Technology (NUST), \\ H-12, ISLAMABAD, PAKISTAN \\ atharkharal@gmail.com
}

\begin{abstract}
This work presents a method of multicriteria decision making using neutrosophic sets. Besides studying some interesting mathematical properties of the method, algorithm viz neut-MCDM is presented. The work also furnishes the fundamentals of neutrosophic set theory succinctly, to provide a first introduction of neutrosophic sets for the MCDM community. To illustrate the computational details, neut-MCDM has been applied to the problem of university faculty selection against a given set of criteria.
\end{abstract}

Keywords: Decision analysis; Theory of DSS, Multiple criteria evaluation; Group decision-making; Neutrosophic sets; Faculty selection;

\section{Introduction}

Since the introduction of fuzzy logic, many systems have been developed in order to deal with approximate and uncertain reasoning: among the latest and most general proposals is neutrosophic logic, introduced by Smarandache [1] as a generalization of fuzzy logic and several related systems. It deals with "the origin, nature and scope of neutralities, as well as their interactions with different ideational spectra". The fundamental thesis of neutrosophy is that every idea has not only a certain degree of truth, as is generally assumed in many-valued logic contexts, but also a falsity degree and an indeterminacy degree that have to be considered independently from each other. In 2003 Atanassov et al. [2] wrote about neutrosophy that

"these ideas, once properly formalized, will have a profound impact on our future dealings with imprecision."

The presence of indeterminacies as an inevitable facet has been realized in a vast array of humanistic and natural systems ranging from weather forecast to sport games and presidential elections. Both Fuzzy Sets and Intuitionistic Fuzzy Sets cannot handle the indeterminate conditions well. Neutrosophy introduces the notion of $\{$ Neut $-A\}$ to represent the indeterminacy.

Neutrosophy has laid the foundation for a whole family of new mathematical theories generalizing both their classical and fuzzy counterparts, such as neutrosophic set theory, neutrosophic probability, neutrosophic statistics and neutrosophic logic. In recent years neutrosophic algebraic structures have also been investigated [3]. On the applications side, the neutrosophic framework has found practical applications in a variety of different

*Corresponding author. Ph: 00923336261309

$\dagger$ A version of this manuscript has also been submitted to arXiv.org on 31 Oct 2011. 
fields, such as relational database systems, semantic web services [4], financial data set detection [5], new economies growth and decline analysis [6], image processing $[7,8,9,10]$ and medical diagnosis [11]. It is clear that neutrosophy still need to be refined and developed from a formal as well as practical/applied points of view [12]. Thus in recent years a number of researchers have shed light on different aspects and applications of neutrosophic sets and logic.

Guo and Cheng [7] employed neutrosophic sets to process the images with noise and proposed a novel neutrosophic approach for image segmentation. The image is described using three membership functions, $T, F$ and $I$. The entropy in neutrosophic domain is defined and employed to evaluate the indeterminacy. The experimental results show that the proposed method not only can perform better on 'clean' images, but also on noisy images. In future this approach can find even more applications in image processing and pattern recognition.

A synergy of Artifical Neural Networks paradigm and Neutrosophic sets has been exploited by Kraipeerapun and Fung in [13]. They presented an ensemble neural network and interval neutrosophic sets approach to the problem of binary classification. A bagging technique is applied to an ensemble of pairs of neural networks created to predict degree of truth membership, indeterminacy membership, and false membership values in the interval neutrosophic sets.

Rivieccio [12] introduces and discusses some basic features of neutrosophic logics from the viewpoint of a family of many-valued systems that can be regarded as generalization of fuzzy logics. The work is a much needed critical examination of Neutrosophic logic and sets. The author points out its many appealing aspects as well as the most controversial ones e.g. indeterminacy degree, and how it can be independent of the truth and falsity degrees. This work encourages to pursue a deeper investigation into neutrosophy that may lead to its proper formalization. Rivieccio presents also future directions for defining suitable neutrosophic propositional connectives while discussing the relationship between neutrosophic logics and other well-known frameworks for reasoning with uncertainty and vagueness, such as intuitionistic and/or interval-valued fuzzy systems and Belnap's four valued-logic.

Quite recently, a new technique of color texture image segmentation that is based on neutrosophic sets and multiresolution wavelet decompositions has been presented by Sengur and Guo in [14]. Neutrosophic sets have helped in this work in two different aspects viz: A fully automatic approach for color texture image segmentation based on neutrosophic set and multiresolution wavelet transformation that enables to segment the color texture image without human intervention. Secondly, adaptive selection on parameters in neutrosophic set that enables to reduce the indeterminacy according to the characteristics of the input image.

In [5] authors have pointed that a combination of statistical testing of audit samples based on Benford's law combined with a neutrosophic reasoning could help the forensic accountant in dealing with misrepresented financial data sets. To the best of our knowledge neutrosophic sets have not been applied to the field of multicriteria decision making. In this paper we present a ground breaking method of multicriteria decision making (MCDM) using Theory of Neutrosophic Sets (TNS).

In the present paper we study an MCDM method using the notion of neutrosophic sets. Organization of the work is as follows: Section 2 explains the fundamentals of neutrosophic set theory in a succinct and coherent manner. Presentation of this section is aimed to furnish all basics of the theory, so that this may serve as a first introduction of neutrosophic 
sets for the MCDM research community. Section 3 presents the main contribution of this work i.e. a method of multicriteria decision making using neutrosophic sets. The section presnts finer mathematical details and properties of the method before giving the algorithm of this method viz. neut-MCDM. Finally Section 4 applies the proposed method to a MCDM problem of university faculty selection against some criteria. This example illustrates the method computationally. The paper concludes with a Conclusion, highlighting the salient contributions and features of this work.

\section{Neutrosophy and Neutrosophic Sets}

Neutrosophic sets and logic are a gradual development from fuzzy logic and its different extensions. Hence, a gradual introduction from fuzzy sets to neutrosophic sets is in point here.

In Fuzzy Set Theory the two-point set of classical truth values $\{0,1\}$ of membership function is replaced by the real unit interval $[0,1]$. Each real value in $[0,1]$ is intended to represent a different degree of truth, ranging from 0 , corresponding to non-membership in classical set theory, to 1 , corresponding to membership. The standard set operations are defined as functions on $[0,1]$, such as if $\mu$ and $\nu$ are fuzzy sets then their intersection and union are given as

$$
\mu \wedge \nu=\min _{x}(\mu(x), \nu(x)) \text { and } \mu \vee \nu=\max _{x}(\mu(x), \nu(x)),
$$

and so on.

Given an element $x$ of universe $X$, whose membership degree is $\mu(x)=t \in[0,1]$, in fuzzy set theory (FST) it is implicitly assumed that it also has a non-membership degree given by $1-t$. This need not hold in general in the so-called intuitionistic fuzzy sets (IFS), a generalization of fuzzy sets introduced by Atanassov [15]. In IFS the non-membership degree of each element is explicitly represented by a second real value $f \in[0,1]$ so that the value of an element $x$ is an ordered pair $\mu(x)=(t, f)$ with $0 \leq t+f \leq 1$. The main novelty of Atanassov's approach is that since one may have $t+f<1$, a certain amount of indeterminacy or incomplete information given as $1-(t+f)$ is permitted.

One may also consider the possibility that $t+f>1$, so that inconsistent beliefs are also allowed, that is, an element may be regarded as both member and non-member at the same time. In this way we obtain a Paraconsistent Set Theory (PST) and corresponding paraconsistent logics. Perhaps the most well known among such logics is Belnap's fourvalued logic.

A neutrosophic set is a generalization of all the previous ones: for instance if we set $\mu(x)=(t, 0,1-t)$ for every element $x$ we obtain the set of membership values corresponding to fuzzy set, if we set $\mu(x)=(t, 1-t-f, f)$ with $t+f \leq 1$ we get IFS and so on.We may then define suitable set operators that generalize the standard operations of fuzzy logic, IFS, PST etc. The main novelty of neutrosophic set is that we do not even assume that the incompleteness or "indeterminacy degree" is always given by $1-(t+f)$.

A formal definition of neutrosophic set depends upon the notion of non-standard real numbers, also known as hyper real numbers, and intervals. In 1960s Abraham Robinson developed the non-standard analysis, a formalization of analysis and a branch of mathematical logic, that rigorously defines the infinitesimals. Informally, an infinitesimal is an infinitely small number. Formally, $x \in \mathbb{R}$ is said to be infinitesimal if and only if for all positive integers $n \in \mathbb{Z}^{+}$one has $|x|<\frac{1}{n}$. Let $\bullet>0$ be such an infinitesimal number. 
Then we consider the non-standard finite numbers given as $1^{+}=1+\bullet$, where " 1 " is its standard part and "•" its non-standard part, and $-0=0-\bullet$, where "0" is its standard part and "•" its non-standard part. Then, we call $]^{-} 0,1^{+}[$a non-standard unit interval.

Definition of neutrosophic set originally given by F. Samarandache is fairly technical and general.

Definition 1. [16] Let $U$ be a space of points (objects), with a generic element in $U$ denoted by $x$. A neutrosophic set $A$ in $U$ is characterized by a truth-membership function $T_{A}$, an indeterminacy-membership function $I_{A}$ and a false-membership function $F_{A}$. $T_{A}(x), I_{A}(x)$ and $F_{A}(x)$ are real standard or non-standard subsets of $]^{-} 0,1^{+}[$. That is

$$
\begin{gathered}
\left.T_{A}: X \rightarrow\right]^{-} 0,1^{+}[, \\
\left.I_{A}: X \rightarrow\right]^{-} 0,1^{+}[, \\
\left.F_{A}: \quad X \rightarrow\right]^{-} 0,1^{+}[.
\end{gathered}
$$

$T_{A}, I_{A}$ and $F_{A}$ are called neutrosophic components. There is no restriction on the sum of $T_{A}(x), I_{A}(x)$ and $F_{A}(x)$, so $^{-} 0 \leq \sup T_{A}(x)+\sup I_{A}(x)+\sup F_{A}(x) \leq 3^{+}$.

It is easy to visualize a neutrosophic set $A$ upon $U$ as an object given as

$$
\left\langle\frac{x}{T_{A}(x), I_{A}(x), F_{A}(x)}\right\rangle=\left\{\frac{x}{T_{A}(x), I_{A}(x), F_{A}(x)}: x \in U\right\},
$$

where $T_{A}(x), I_{A}(x), F_{A}(x)$ are subintervals or union of subintervals of $I=[0,1]$. Thus a neutrosophic set $A=\left\langle\frac{x}{T_{A}(x), I_{A}(x), F_{A}(x)}\right\rangle$ in $U$ can be identified to an ordered triplet $<T_{A}, I_{A}, F_{A}>$ in $I^{X} \times I^{X} \times I^{X}$ or to an element in $(I \times I \times I)^{X}$.

The element $\frac{x}{t, i, f}$ belongs to $A$ in the following way: it is $t \%$ true, $i \%$ indeterminate, and $f \%$ false, where $t$ varies in $T_{A}, i$ varies in $I_{A}$, and $f$ varies in $F_{A}$. Statically, $T_{A}, I_{A}$ and $F_{A}$ are membership sets, but dynamically $T_{A}, I_{A}$ and $F_{A}$ are functions depending on known and/or unknown parameters. Neutrosophic components may overlap, as well. We give an example to further clarify the notion of neutrosophic set:

Example 2. Suppose we have a claim $p$ given as:

$$
p=\text { Rain is expected tomorrow. }
$$

Two meteorologists have their respective opinions about the truthhood of $p$. One expert puts the truth degree of $p$ between 0 and 0.4 , the second between 0.6 and 0.8 . Both the experts put the uncertainty or hesitation as somewhere between 0.1 and 0.3 and 0.2 to 0.4. Similarly they are definite that $p$ would not hold to the degree between 0.5 to 0.6 and 0.3 and 0.5 , respectively. Both expert opinions now may be represented by neutrosophic sets $A$ and $B$ as:

$$
A=\left\{\frac{p}{[0,0.4],[0.1,0.3],[0.5,0.6]}\right\}, \quad B=\left\{\frac{p}{[0.6,0.8],[0.2,0.4],[0.3,0.5]}\right\} .
$$

Neutrosophy permits us to even represent both expert opinion under one neutrosophic set, say $C$, as:

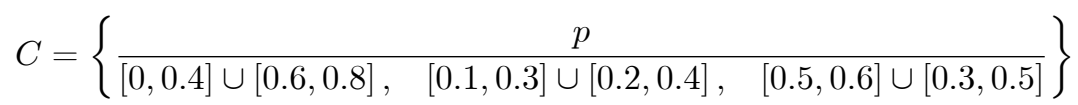


This characteristic of neutrosophic sets of representing even different and distinct pieces of information with uniform framework is peculiar to this theory vis-a-vis Fuzzy Sets and Intuitionistic Fuzzy Sets.

The sets $T_{A}, I_{A}$ and $F_{A}$ are not necessarily intervals, and may be any real sub-unitary subsets: discrete or continuous; single-element, finite, or countable or uncountable infinite; union or intersection of various subsets; etc. Such a liberty enables the NST to deal with information coming from different, possibly conflicting sources, even. Intuitively, the set $I \subseteq[0,1]$ may represent not only indeterminacy but also vagueness, uncertainty, imprecision, error, etc.

In this paper we are concerned with neutrosophic sets whose $T_{A}, I_{A}$ and $F_{A}$ values are single points in $[0,1]$ instead of subintervals/subsets in $[0,1]$. Consequently, in the sequel, we shall write an element in neutrosophic set $A$ simply as $\frac{x}{T_{A}, I_{A}, F_{A}}$ instead of writing it as

$$
\left\{\frac{x}{t, i, f} \mid t \in T_{A}(x), i \in I_{A}(x), f \in F_{A}(x)\right\} .
$$

An example may further clarify the point:

Example 3. Consider a decision making problem where 5 judges out of 10 say "yes" to some alternative $a, 3$ say "no" and 2 are undecided. But out of the same judges, 3 say "yes" for another alternative $b, 5$ abstain, and 2 say "no" for $b$. We may represent this decision making situation as the neutrosophic set $\left\{\frac{a}{0.5,0.2,0.3}, \frac{b}{0.3,0.5,0.2}\right\}$.

In Neutrosophy, every object has not only a certain degree of truth, but also a falsity degree and an indeterminacy degree that have to be considered independently from each other. Thus, a theory, event, concept, or entity, $\{A\}$ is considered with its opposite $\{A n t i-A\}$ and the neutrality $\{$ Neut $-A\}$. $\{$ Neut $-A\}$ is neither $\{A\}$ nor $\{A n t i-A\}$. The $\{$ Neut $-A\}$ and $\{A n t i-A\}$ are referred to as $\{$ Non $-A\}$, symbolically

$$
\{\text { Non }-A\}=\{\text { Neut }-A\} \cup\{\text { Anti }-A\} .
$$

According to this theory, every idea $\{A\}$ is neutralized and balanced by $\{A n t i-A\}$ and $\{$ Non $-A\}$.

Now we give the basic definitions of operations on neutrosophic sets [17]. We will concentrate on the case when the neutrosophic components are real values within unit interval instead of subintervals or subsets of the unit interval.

Definition 4. Let $U$ be a universe and $A$ and $B$ are neutrosophic sets upon $U$. For $\frac{x}{T_{A}, I_{A}, F_{A}} \in A$ and $\frac{x}{T_{B}, I_{B}, F_{B}} \in B$ we define

1. Complement of $A$ as

$$
A^{c}=\left\{\frac{x}{T, I, F} \mid T=1-T_{A}, I=1-I_{A}, F=1-F_{A}\right\},
$$

2. Intersection of $A$ and $B$ as

$$
A \cap B=\left\{\frac{x}{T, I, F} \mid T=T_{A} T_{B}, I=I_{A} I_{B}, F=F_{A} F_{B}\right\},
$$


3. Union of $A$ and $B$ as

$$
A \cup B=\left\{\frac{x}{T, I, F} \mid T=T_{A}+T_{B}-T_{A} T_{B}, I=I_{A}+I_{B}-I_{A} I_{B}, F=F_{A}+F_{B}-F_{A} F_{B}\right\},
$$

4. Difference of $A$ and $B$ as

$$
A \backslash B=\left\{\frac{x}{T, I, F} \mid T=T_{A}-T_{A} T_{B}, I=I_{A}-I_{A} I_{B}, F=F_{A}-F_{A} F_{B}\right\},
$$

5. Cartesian product of $A$ and $B$ as

$$
A \times B=\left\{\left(\frac{x}{T_{A}, I_{A}, F_{A}}, \frac{y}{T_{B}, I_{B}, F_{B}}\right) \mid \frac{x}{T_{A}, I_{A}, F_{A}} \in A, \frac{y}{T_{B}, I_{B}, F_{B}} \in B\right\},
$$

6. $A$ is subset of $B$ as

$$
A \subseteq B \Longleftrightarrow \forall \frac{x}{T_{A}, I_{A}, F_{A}} \in A, \frac{y}{T_{B}, I_{B}, F_{B}} \in B, T_{A} \leq T_{B} \text { and } F_{A} \geq F_{B} .
$$

The set of operators given above are not unique for definition of respective operations between neutrosophic sets. Some other schemes of operators may also be seen in literature. In this paper we shall limit ourselves to only above-defined operations.

Example 5. Let $I=[0,1]$ be the universal set and $A, B$ neutrosophic sets given as:

$$
\begin{aligned}
& A=\left\{\frac{x}{\frac{1}{5} e^{\frac{5}{3}-\frac{10}{3} x}, \frac{1}{10} e^{5-10 x},-\frac{1}{10 e^{5 x-\frac{3}{2}}-10}}: x \in[0,1]\right\} \\
& B=\left\{\frac{x}{\frac{3}{10}(2+\sin (10 x)), \frac{1}{1+\tan (x)}, x-x^{2}}: x \in[0,1]\right\} .
\end{aligned}
$$


Then their graphical representation and of their different operations is given as:

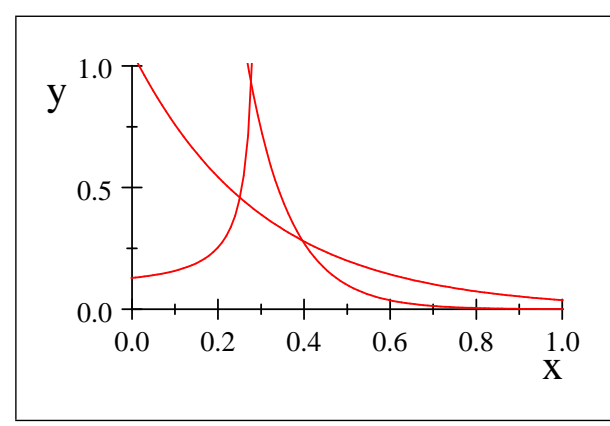

Neutrosophic Set A
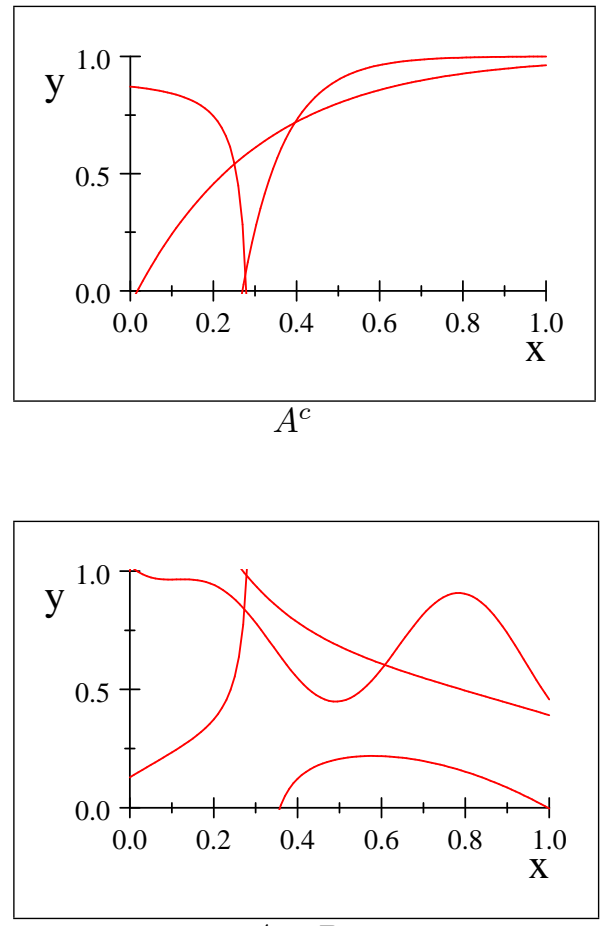

$A \cup B$

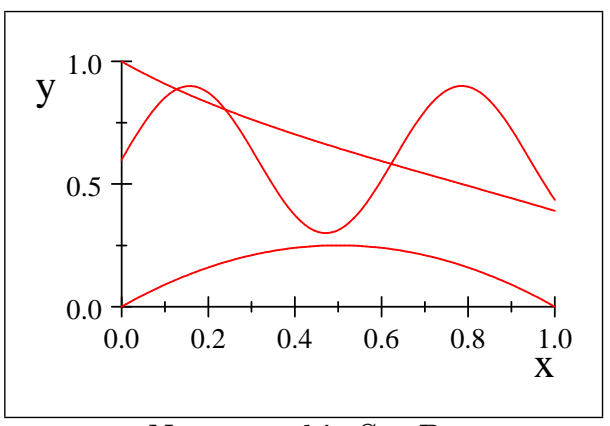

Neutrosophic Set B
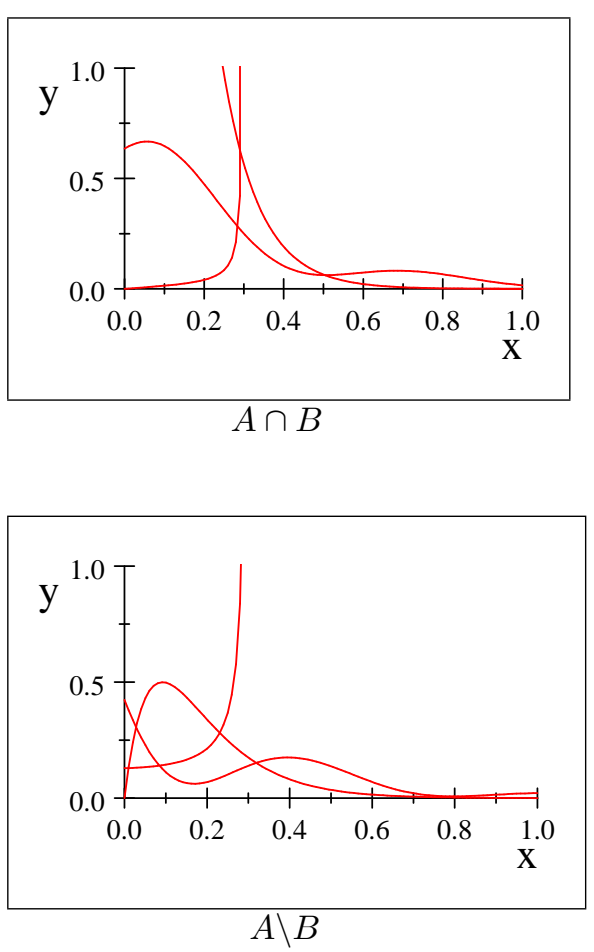

Example 6. Let $U=\{a, b, c\}$ and $A, B$ and $C$ are neutrosophic sets given as:

$$
\begin{aligned}
A & =\left\{\frac{a}{0.4,0.3,0.5}, \frac{b}{0.5,0.3,0.1}, \frac{c}{0.2,0.5,0.9}\right\}, \\
B & =\left\{\frac{a}{0.3,0.5,0.5}, \frac{b}{0.1,0.4,0.6}, \frac{c}{0.5,0.5,0.5}\right\}, \\
C & =\left\{\frac{a}{0.4,0.3,0.2}, \frac{b}{0.6,0.3,0.1}, \frac{c}{0.4,0.3,0.8}\right\} .
\end{aligned}
$$


Then we compute

$$
\begin{aligned}
& A^{c}=\left\{\frac{a}{0.6,0.7,0.5}, \frac{b}{0.5,0.7,0.9}, \frac{c}{0.8,0.5,0.1}\right\}, \\
& A \cap B=\left\{\frac{a}{0.12,0.15,0.25}, \frac{b}{0.05,0.12,0.06}, \frac{c}{0.1,0.25,0.45}\right\}, \\
& A \cup B=\left\{\frac{a}{0.58,0.65,0.75}, \frac{b}{0.55,0.58,0.64}, \frac{c}{0.6,0.75,0.95}\right\}, \\
& A \backslash B=\left\{\frac{a}{0.28,0.15,0.25}, \frac{b}{0.45,0.18,0.04}, \frac{c}{0.1,0.25,0.45}\right\},
\end{aligned}
$$

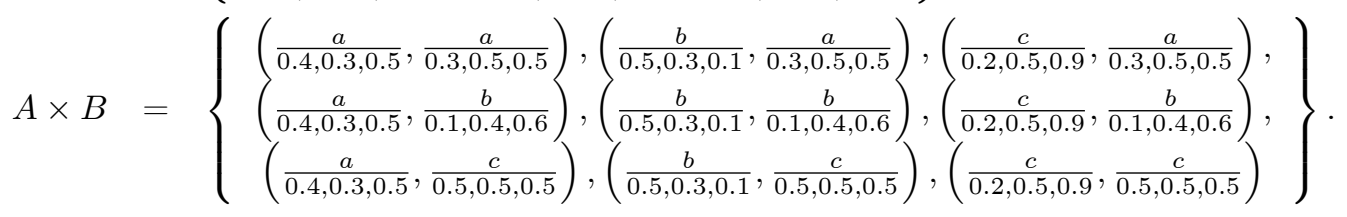

Moreover we have

$$
A \nsubseteq B \text { and } A \subseteq C .
$$

There is no notion of order embedded into the notion of neutrosophic set. But supposing such an order over universal set $U$ facilitates matrix notation of different neutrosophic sets arising in a situation. For example by supposing that first column corresponds to $a$, second to $b$ and third to $c$, the neutrosophic sets given in Example 6 may also be written as:

$$
\begin{aligned}
& A \\
& B \\
& C
\end{aligned} \quad\left[\begin{array}{ccc}
a & b & c \\
{[0.4,0.3,0.5]} & {[0.5,0.3,0.1]} & {[0.2,0.5,0.9]} \\
{[0.3,0.5,0.5]} & {[0.1,0.4,0.6]} & {[0.5,0.5,0.5]} \\
{[0.4,0.3,0.2]} & {[0.6,0.3,0.1]} & {[0.4,0.3,0.8]}
\end{array}\right]
$$

Use of square brackets as elements of above matrix denotes the vector nature of entries i.e. $[T, I, F]$.

\section{Multicriteria Decision Making Based on Neutrosophic Sets}

This section presents a new method for handling neutrosophic multicriteria decisionmaking problems, where the characteristics of the alternatives are represented by neutrosophic sets. Suppose there exists a set of alternatives $A=\left\{A_{1}, A_{2}, \ldots, A_{m}\right\}$ which consists of $m$ non-dominated decision-making alternatives from which the most preferred alternative is to be selected. Each alternative is assessed on $n$ different criteria. Denote the set of all criteria $C=\left\{C_{1}, C_{2}, \ldots, C_{n}\right\}$. Assume that $T_{i j}, I_{i j}$ and $F_{i j}$ are the degrees of membership, ideterminacy and non-membership of the alternative $A_{i} \in A$ satisfying the criterion $C_{j} \in C$, respectively, where $0 \leq T_{i j} \leq 1,0 \leq I_{i j} \leq 1,0 \leq F_{i j} \leq 1$ and $0 \leq T_{i j}+I_{i j}+F_{i j} \leq 3$. In other words, the evaluation of the alternative $A_{i}$ with respect to the criterion $C_{j}$ is a neutrosophic set. The neutrosophic indices $I_{i j}$ is such that the larger $I_{i j}$ the higher a hesitation margin of the decision maker of the alternative $A_{i}$ with 
respect to the criterion $C_{j}$ whose intensity is given by $T_{i j}$. The decision matrix $D$ is given in the following form:

$$
\begin{gathered}
A_{1} \\
A_{2} \\
\vdots \\
A_{m}
\end{gathered}\left[\begin{array}{cccc}
\left(T_{11}, I_{11}, F_{11}\right) & C_{2} & \cdots & C_{n} \\
\left(T_{21}, I_{21}, F_{21}\right) & \left(T_{12}, I_{12}, F_{12}\right) & \cdots & \left(T_{1 n}, I_{1 n}, F_{1 n}\right) \\
\vdots & \vdots & \ddots & \left(T_{2 n}, I_{2 n}, F_{2 n}\right) \\
\left(T_{m 1}, I_{m 1}, F_{m 1}\right) & \left(T_{m 2}, I_{m 2}, F_{m 2}\right) & \cdots & \left(T_{m n}, I_{m n}, F_{m n}\right)
\end{array}\right]
$$

where the characteristics of the alternative $A_{i}$ are given by the neutrosophic set shown as:

$$
\begin{aligned}
A_{i} & =\left\{\frac{C_{1}}{T_{i 1}, I_{i 1}, F_{i 1}}, \frac{C_{2}}{T_{i 2}, I_{i 2}, F_{i 2}}, \ldots, \frac{C_{n}}{T_{i n}, I_{i n}, F_{i n}}\right\} \\
& =\left\{\frac{C_{j}}{T_{i j}, I_{i j}, F_{i j}} \mid C_{j} \in C\right\}
\end{aligned}
$$

where $1 \leq i \leq m$.

Neutrosophic indices allow us to calculate the best final result (and the worst one) we can expect in a process leading to a final optimal decision. During the process the decision-maker can change his evaluations in the following way. He can increase his evaluation by adding the value of the neutrosophic index. So in fact his evaluation lies in the closed interval $\left[T_{i j}^{l}, T_{i j}^{u}\right]=\left[\min \left(\frac{T_{i j}+I_{i j}}{2}, \frac{1-F_{i j}+I_{i j}}{2}\right), \max \left(\frac{T_{i j}+I_{i j}}{2}, \frac{1-F_{i j}+I_{i j}}{2}\right)\right]$, where $T_{i j}^{l}=\min \left(\frac{T_{i j}+I_{i j}}{2}, \frac{1-F_{i j}+I_{i j}}{2}\right)$, and $T_{i j}^{u}=\max \left(\frac{T_{i j}+I_{i j}}{2}, \frac{1-F_{i j}+I_{i j}}{2}\right)$. Obviously, $0 \leq$ $T_{i j}^{l}+T_{i j}^{u} \leq 2$ for all $A_{i} \in A$ and $C_{j} \in C$.

We can present $A_{i}$ by another form as follows for the sake of performing the decisionmaker's evaluation more directly,

$$
A_{i}=\left\{\left\langle C_{1},\left[T_{i 1}^{l}, T_{i 1}^{u}\right]\right\rangle,\left\langle C_{2},\left[T_{i 2}^{l}, T_{i 2}^{u}\right]\right\rangle, \cdots,\left\langle C_{n},\left[T_{i n}^{l}, T_{i n}^{u}\right]\right\rangle\right\}
$$

Assume that there is a decision-maker who wants to choose an alternative which satisfies the criteria $C_{j}, C_{k}, \ldots, C_{p}$ or which satisfies the criteria $C_{s}$. This decision-maker's requirement is represented by the following expression:

$$
C_{j} A N D C_{k} A N D \ldots A N D C_{p} O R C_{s}
$$

Conveniently, suppose $E\left(A_{i}\right)$ is the evaluation function of alternative $A_{i}$, and which can be expressed as $E\left(A_{i}\right)=\left[T_{A_{i}}^{l}, T_{A_{i}}^{u}\right]$. In this case, the degrees to which the alternative $A_{i}$ satisfies and does not satisfy the decision-maker's requirement can be measured by the evaluation function $E$,

$$
\begin{aligned}
E\left(A_{i}\right) & =\left[T_{i j}^{l}, T_{i j}^{u}\right] \wedge\left[T_{i k}^{l}, T_{i k}^{u}\right] \wedge \ldots \wedge\left[T_{i p}^{l}, T_{i p}^{u}\right] \vee\left[T_{i s}^{l}, T_{i s}^{u}\right] \\
& =\left[\min \left\{T_{i j}^{l}, T_{i k}^{l}, \ldots, T_{i p}^{l}\right\}, \min \left\{T_{i j}^{u}, T_{i k}^{u}, \ldots, T_{i p}^{u}\right\}\right] \vee\left[T_{i s}^{l}, T_{i s}^{u}\right] \\
& =\left[\max \left\{\min \left\{T_{i j}^{l}, T_{i k}^{l}, \ldots, T_{i p}^{l}\right\}, T_{i s}^{l}\right\}, \max \left\{\min \left\{T_{i j}^{u}, T_{i k}^{u}, \ldots, T_{i p}^{u}\right\}, T_{i s}^{u}\right\}\right] \\
& =\left[T_{A_{i}}^{l}, T_{A_{i}}^{u}\right],
\end{aligned}
$$


where $\wedge$ and $\vee$ denote the minimum operator and the maximum operator of NST, respectively; $1 \leq i \leq m$.

Let $A_{i}=\left[\min \left(\frac{T_{i j}+I_{i j}}{2}, \frac{1-F_{i j}+I_{i j}}{2}\right), \max \left(\frac{T_{i j}+I_{i j}}{2}, \frac{1-F_{i j}+I_{i j}}{2}\right)\right]$, where $\min \left(\frac{T_{i j}+I_{i j}}{2}, \frac{1-F_{i j}+I_{i j}}{2}\right) \in$ $[0,1], \max \left(\frac{T_{i j}+I_{i j}}{2}, \frac{1-F_{i j}+I_{i j}}{2}\right) \in[0,1], \min \left(\frac{T_{i j}+I_{i j}}{2}, \frac{1-F_{i j}+I_{i j}}{2}\right)+\max \left(\frac{T_{i j}+I_{i j}}{2}, \frac{1-F_{i j}+I_{i j}}{2}\right) \leq$ 1. The score of an alternative $A_{i}$ can be evaluated by the score function $S$ shown as

$$
\begin{aligned}
S\left(A_{i}\right) & =2\left(T_{A_{i}}^{u}-T_{A_{i}}^{l}\right) \\
& =2\left(\max \left(\left(\frac{T_{A_{i}}+I_{A_{i}}}{2}\right),\left(\frac{1-F_{A_{i}}+I_{A_{i}}}{2}\right)\right)-\min \left(\left(\frac{T_{A_{i}}+I_{A_{i}}}{2}\right),\left(\frac{1-F_{A_{i}}+I_{A_{i}}}{2}\right)\right)\right)
\end{aligned}
$$

Next, we define an accuracy function $H$ to evaluate the degree of accuracy of neutrosophic elements as follows:

$$
\begin{aligned}
H\left(A_{i}\right) & =\frac{1}{2}\left(T_{A_{i}}^{l}+T_{A_{i}}^{u}\right) \\
& =\frac{1}{2}\left[\min \left(\left(\frac{T_{A_{i}}+I_{A_{i}}}{2}\right),\left(\frac{1-F_{A_{i}}+I_{A_{i}}}{2}\right)\right)+\max \left(\left(\frac{T_{A_{i}}+I_{A_{i}}}{2}\right),\left(\frac{1-F_{A_{i}}+I_{A_{i}}}{2}\right)\right)\right]
\end{aligned}
$$

Proposition 7. Let $\left(T_{A_{i}}, I_{A_{i}}, F_{A_{i}}\right)$ be neutrosophic components of an element $A_{i}$ in a neutrosophic set $A$ and

(1) $S\left(A_{i}\right)$ be scoring function defined as

$S\left(A_{i}\right)=2\left(\max \left(\left(\frac{T_{A_{i}}+I_{A_{i}}}{2}\right),\left(\frac{1-F_{A_{i}}+I_{A_{i}}}{2}\right)\right)-\min \left(\left(\frac{T_{A_{i}}+I_{A_{i}}}{2}\right),\left(\frac{1-F_{A_{i}}+I_{A_{i}}}{2}\right)\right)\right)$,

then we have

$$
0 \leq S\left(A_{i}\right) \leq 1
$$

(2) $H\left(A_{i}\right)$ be scoring function defined as

$$
H\left(A_{i}\right)=\frac{1}{2}\left[\min \left(\left(\frac{T_{A_{i}}+I_{A_{i}}}{2}\right),\left(\frac{1-F_{A_{i}}+I_{A_{i}}}{2}\right)\right)+\max \left(\left(\frac{T_{A_{i}}+I_{A_{i}}}{2}\right),\left(\frac{1-F_{A_{i}}+I_{A_{i}}}{2}\right)\right)\right],
$$

then we have

$$
0 \leq H\left(A_{i}\right) \leq 1
$$

Proof. (1) By definition

$\left.T_{A_{i}}^{l}=\min \left(\left(\frac{T_{A_{i}}+I_{A_{i}}}{2}\right),\left(\frac{1-F_{A_{i}}+I_{A_{i}}}{2}\right)\right)\right) \leq \max \left(\left(\frac{T_{A_{i}}+I_{A_{i}}}{2}\right),\left(\frac{1-F_{A_{i}}+I_{A_{i}}}{2}\right)\right)=T_{A_{i}}^{u}$. 
For the case when $T_{A_{i}}^{l}=\left(\frac{T_{A_{i}}+I_{A_{i}}}{2}\right)$ and $T_{A_{i}}^{u}=\left(\frac{1-F_{A_{i}}+I_{A_{i}}}{2}\right)$ we have

$$
\begin{aligned}
2\left(T_{A_{i}}^{u}-T_{A_{i}}^{l}\right) & \geq 0 \\
2\left(\left(\frac{1-F_{A_{i}}+I_{A_{i}}}{2}\right)-\left(\frac{T_{A_{i}}+I_{A_{i}}}{2}\right)\right) & \geq 0 \\
\left(\frac{1-F_{A_{i}}+I_{A_{i}}}{2}\right) & \geq\left(\frac{T_{A_{i}}+I_{A_{i}}}{2}\right) \\
1-F_{A_{i}}+I_{A_{i}} & \geq T_{A_{i}}+I_{A_{i}} \\
T_{A_{i}}+F_{A_{i}} & \leq 1
\end{aligned}
$$

This gives

$$
\begin{aligned}
S\left(A_{i}\right) & =2\left(T_{A_{i}}^{u}-T_{A_{i}}^{l}\right) \\
& =2\left(\left(\frac{1-F_{A_{i}}+I_{A_{i}}}{2}\right)-\left(\frac{T_{A_{i}}+I_{A_{i}}}{2}\right)\right) \\
& =2\left(\frac{1}{2}-\frac{1}{2} T_{A_{i}}-\frac{1}{2} F_{A_{i}}\right) \\
& =1-T_{A_{i}}-F_{A_{i}} \leq 1 \quad \text { by }(1) .
\end{aligned}
$$

Furthermore as $T_{A_{i}}, F_{A_{i}} \in[0,1]$, we have $S\left(A_{i}\right)=2\left(T_{A_{i}}^{u}-T_{A_{i}}^{l}\right) \in[0,1]$. have

Similarly, for the other case i.e. when $T_{A_{i}}^{l}=\left(\frac{1-F_{A_{i}}+I_{A_{i}}}{2}\right)$ and $T_{A_{i}}^{u}=\left(\frac{T_{A_{i}}+I_{A_{i}}}{2}\right)$ we

$$
\begin{aligned}
T_{A_{i}}^{l} & \leq T_{A_{i}}^{u} \\
& \Rightarrow 2\left(T_{A_{i}}^{u}-T_{A_{i}}^{l}\right) \geq 0 \\
& \Rightarrow 2\left(\left(\frac{T_{A_{i}}+I_{A_{i}}}{2}\right)-\left(\frac{1-F_{A_{i}}+I_{A_{i}}}{2}\right)\right) \geq 0 \\
T_{A_{i}}+I_{A_{i}} & \geq 1-F_{A_{i}}+I_{A_{i}} \\
T_{A_{i}}+F_{A_{i}} & \geq 1
\end{aligned}
$$

And in this case

$$
\begin{aligned}
S\left(A_{i}\right) & =2\left(T_{A_{i}}^{u}-T_{A_{i}}^{l}\right)=2\left(\left(\frac{T_{A_{i}}+I_{A_{i}}}{2}\right)-\left(\frac{1-F_{A_{i}}+I_{A_{i}}}{2}\right)\right) \\
& =2\left(\frac{1}{2} F_{A_{i}}+\frac{1}{2} T_{A_{i}}-\frac{1}{2}\right)=T_{A_{i}}+F_{A_{i}}-1 \\
& \Rightarrow S\left(A_{i}\right) \in[0,1] \quad \text { by }(2) .
\end{aligned}
$$

Hence the result.

(2)For the case when $T_{A_{i}}^{l}=\left(\frac{T_{A_{i}}+I_{A_{i}}}{2}\right)$ and $T_{A_{i}}^{u}=\left(\frac{1-F_{A_{i}}+I_{A_{i}}}{2}\right)$ we have

$$
\begin{aligned}
H\left(A_{i}\right) & =\frac{\left(\frac{T_{A_{i}}+I_{A_{i}}}{2}\right)+\left(\frac{1-F_{A_{i}}+I_{A_{i}}}{2}\right)}{2} \\
& =\frac{1}{2} I_{A_{i}}-\frac{1}{4} F_{A_{i}}+\frac{1}{4} T_{A_{i}}+\frac{1}{4}
\end{aligned}
$$


By examining the extreme possible values of $T_{A_{i}}, I_{A_{i}}$ and $F_{A_{i}}$ we have

$$
\begin{aligned}
& {\left[\frac{1}{2} I_{A_{i}}-\frac{1}{4} F_{A_{i}}+\frac{1}{4} T_{A_{i}}+\frac{1}{4}\right]_{T_{A_{i}}=0, I_{A_{i}}=0, F_{A_{i}}=0}=\frac{1}{4},} \\
& {\left[\frac{1}{2} I_{A_{i}}-\frac{1}{4} F_{A_{i}}+\frac{1}{4} T_{A_{i}}+\frac{1}{4}\right]_{T_{A_{i}}=1, I_{A_{i}}=0, F_{A_{i}}=0}=\frac{1}{2}} \\
& {\left[\frac{1}{2} I_{A_{i}}-\frac{1}{4} F_{A_{i}}+\frac{1}{4} T_{A_{i}}+\frac{1}{4}\right]_{T_{A_{i}}=1, I_{A_{i}}=1, F_{A_{i}}=0}=1} \\
& {\left[\frac{1}{2} I_{A_{i}}-\frac{1}{4} F_{A_{i}}+\frac{1}{4} T_{A_{i}}+\frac{1}{4}\right]_{T_{A_{i}}=1, I_{A_{i}}=1, F_{A_{i}}=1}=\frac{3}{4}} \\
& {\left[\frac{1}{2} I_{A_{i}}-\frac{1}{4} F_{A_{i}}+\frac{1}{4} T_{A_{i}}+\frac{1}{4}\right]_{T_{A_{i}}=0, I_{A_{i}}=1, F_{A_{i}}=1}=\frac{1}{2}} \\
& {\left[\frac{1}{2} I_{A_{i}}-\frac{1}{4} F_{A_{i}}+\frac{1}{4} T_{A_{i}}+\frac{1}{4}\right]_{T_{A_{i}}=0, I_{A_{i}}=0, F_{A_{i}}=1}=0}
\end{aligned}
$$

Hence the result.

We know that the value of $I$ denotes a measure of non-determinacy. The larger it is, the higher a hesitation margin of the decision-maker. So the larger the value of $H\left(A_{i}\right)$, the more the degree of accuracy of an element $A_{i}$ in the neutrosophic set $A$. Now we want to make use of the two functions $S$ and $H$ to establish a function, which can measure the degree to which alternatives satisfy the decision-maker's requirement. But if we simply add them up directly, the value of $F_{A_{i}}$ will be deleted. So based on the score function $S$ and the accuracy function $H$, the degree of suitability to which the alternative $A_{i}$ satisfies the decision-maker's requirement can be measured as follows:

$$
W\left(E\left(A_{i}\right)\right)=\left(S\left(E\left(A_{i}\right)\right)\right)^{2}-\left(\frac{1-H\left(E\left(A_{i}\right)\right)}{2}\right)
$$

The coeeficients in $W\left(E\left(A_{i}\right)\right)$ have been chosen so that $W\left(E\left(A_{i}\right)\right) \in[0,1]$. Moreover, for calculation purposes we may find another expression for the weight function as:

$$
\begin{aligned}
W\left(E\left(A_{i}\right)\right) & =\left(S\left(E\left(A_{i}\right)\right)\right)^{2}-\left(\frac{1-H\left(E\left(A_{i}\right)\right)}{2}\right) \\
& =\left(2\left(T_{A_{i}}^{u}-T_{A_{i}}^{l}\right)\right)^{2}-\left(\frac{\left.1-\frac{T_{A_{i}}^{l}+T_{A_{i}}^{u}}{2}\right)}{2}\right) \\
& =4\left(T_{A_{i}}^{u}-T_{A_{i}}^{l}\right)^{2}-\frac{1}{2}\left(1-\frac{1}{2} T_{A_{i}}^{u}-\frac{1}{2} T_{A_{i}}^{l}\right) \\
& =4\left(\left(T_{A_{i}}^{l}\right)^{2}-2 T_{A_{i}}^{l} T_{A_{i}}^{u}+\left(T_{A_{i}}^{u}\right)^{2}\right)-\frac{1}{2}\left(1-\frac{1}{2} T_{A_{i}}^{u}-\frac{1}{2} T_{A_{i}}^{l}\right) \\
& =4\left(T_{A_{i}}^{l}\right)^{2}+4\left(T_{A_{i}}^{u}\right)^{2}-8 T_{A_{i}}^{l} T_{A_{i}}^{u}-\frac{1}{2}-\frac{1}{4} T_{A_{i}}^{u}-\frac{1}{4} T_{A_{i}}^{l}
\end{aligned}
$$

The larger the value of $W\left(E\left(A_{i}\right)\right)$, the more the suitability to which the alternative $A_{i}$ satisfies the decision-maker's requirement, where $1 \leq i \leq m$. 
Previously, we assumed that all criteria have the same degree of importance. However, if we can allow each criterion to have a different degree of importance, then there is room for more flexibility. A weighted technique for handling multicriteria fuzzy decisionmaking problems may be envisaged, but assuming that the degree of importance of the criteria entered by the decision-maker are constant, is hard to do in reality. So in this paper we assume that $\widehat{T}_{j}, \widehat{I}_{j}$ and $\widehat{F}_{j}$ are the degrees of membership, ideterminacy and non-membership of the criteria $C_{j} \in C$ to the vague concept "importance of criterion", respectively, where $0 \leq \widehat{T}_{j} \leq 1,0 \leq \widehat{I}_{j} \leq 1,0 \leq \widehat{F}_{j} \leq 1$ and $0 \leq \widehat{T}_{j}+\widehat{I}_{j}+\widehat{F}_{j} \leq 3$.

The neutrosophic index $\widehat{I}_{j}$ are such that the larger $\widehat{I}_{j}$ the higher a hesitation margin of decision-maker as to the "importance of the criteria" $C_{j}$ whose intensity is given by $\widehat{T}_{j}$. Neutrosophic indices allow us to calculate the biggest weight (and the smallest one) we can expect in a process leading to a final decision. During the process the decision-maker can change his evaluating weights in the following way. He can increase his evaluating weights by adding the value of the neutrosophic index. So in fact his weight lies in the closed interval $\left[\omega_{j}^{l}, \omega_{j}^{u}\right]=\left[\min \left(\frac{\widehat{T}_{j}+\widehat{I}_{j}}{2}, \frac{1-\widehat{F}_{j}+\widehat{I}_{j}}{2}\right), \max \left(\frac{\widehat{T}_{j}+\widehat{I}_{j}}{2}, \frac{1-\widehat{F}_{j}+\widehat{I}_{j}}{2}\right)\right]$. Obviously, $0 \leq \omega_{j}^{l} \leq \omega_{j}^{u} \leq 1$ for each criterion $C_{j} \in C$.

Assume that there is a decision-maker who wants to choose an alternative which satisfies the criteria $C_{j}, C_{k}, \ldots, C_{p}$ or which satisfies the criteria $C_{s}$. This decisionmaker's requirement can be represented by (1). The degree of importance of the criteria $C_{j}, C_{k}, \ldots, C_{p}$ entered by the decision-maker are $\omega_{j}, \omega_{k}, \ldots, \omega_{p}$, respectively, where $\omega_{j}^{l} \leq \omega_{j} \leq \omega_{j}^{u}, \omega_{k}^{l} \leq \omega_{k} \leq \omega_{k}^{u}, \ldots, \omega_{p}^{l} \leq \omega_{p} \leq \omega_{p}^{u}$ and $\omega_{j}+\omega_{k}+\ldots+\omega_{p}=1$. Let

$$
\begin{aligned}
T\left(A_{i}\right) & =H\left(\left[\widehat{T}_{i j}^{l}, \widehat{T}_{i j}^{u}\right]\right) * \omega_{j}+H\left(\left[\widehat{T}_{i k}^{l}, \widehat{T}_{i k}^{u}\right]\right) * \omega_{k}+\cdots+H\left(\left[\widehat{T}_{i p}^{l}, \widehat{T}_{i p}^{u}\right]\right) * \omega_{p}(2) \\
& =\sum_{r=j}^{p} H\left(\left[\widehat{T}_{i r}^{l}, \widehat{T}_{i r}^{u}\right]\right) * \omega_{r} \\
W\left(A_{i}\right) & =S\left(\left[\widehat{T}_{i j}^{l}, \widehat{T}_{i j}^{u}\right]\right) * \omega_{j}+S\left(\left[\widehat{T}_{i k}^{l}, \widehat{T}_{i k}^{u}\right]\right) * \omega_{k}+\cdots+S\left(\left[\widehat{T}_{i p}^{l}, \widehat{T}_{i p}^{u}\right]\right) * \omega_{p},(3) \\
& =\sum_{s=j}^{p} S\left(\left[\widehat{T}_{i s}^{l}, \widehat{T}_{i s}^{u}\right]\right) * \omega_{s}
\end{aligned}
$$

where $1 \leq i \leq m$. Then the degree of suitability that the alternative $A_{i}$ satisfies the 
decision-maker's requirement can be measured by the following function:

$$
\begin{aligned}
& R\left(A_{i}\right)=\max \left\{\left(W\left(A_{i}\right)\right)^{2}-\left(\frac{1-T\left(A_{i}\right)}{2}\right),\left(S\left(\left[\widehat{T}_{i s}^{l}, \widehat{T}_{i s}^{u}\right]\right)\right)^{2}-\frac{1-H\left(\left[\widehat{T}_{i s}^{l}, \widehat{T}_{i s}^{u}\right]\right)}{2}(\xi)\right.\left\{\begin{array}{c}
\left(\sum_{s=j}^{p} S\left(\left[\widehat{T}_{i s}^{l}, \widehat{T}_{i s}^{u}\right]\right) * \omega_{s}\right)^{2}-\frac{1-\sum_{r=j}^{p} H\left(\left[\widehat{T}_{i r}^{l}, \widehat{T}_{i r}^{u}\right]\right) * \omega_{r}}{2}, \\
\left(S\left(\left[\widehat{T}_{i s}^{l}, \widehat{T}_{i s}^{u}\right]\right)\right)^{2}-\frac{1-H\left(\left[\widehat{T}_{i s}^{l}, \widehat{T}_{i s}^{u}\right]\right)}{2}
\end{array}\right\} \\
&= \max \left\{\begin{array}{c}
\left(\sum_{s=j}^{p} S\left(\left[\widehat{T}_{i s}^{l}, \widehat{T}_{i s}^{u}\right]\right) * \omega_{s}\right)^{2}-\frac{1-\sum_{r=j}^{p} H\left(\left[\widehat{T}_{i r}^{l}, \widehat{T}_{i r}^{u}\right]\right) * \omega_{r}}{2}, \\
\left(2\left(\widehat{T}_{A_{i}}^{u}-\widehat{T}_{A_{i}}^{l}\right)\right)^{2}-\left(\frac{\left.1-\frac{\widehat{T}_{A_{i}}^{l}+\widehat{T}_{A_{i}}^{u}}{2}\right)}{2}\right)
\end{array}\right\} \\
&= \max \left\{\begin{array}{c}
\left(\sum_{s=j}^{p} S\left(\left[\widehat{T}_{i s}^{l}, \widehat{T}_{i s}^{u}\right]\right) * \omega_{s}\right)^{2}-\frac{1-\sum_{r=j}^{p} H\left(\left[\widehat{T}_{i r}^{l}, \widehat{T}_{i r}^{u}\right]\right) * \omega_{r}}{2}, \\
4\left(\widehat{T}_{A_{i}}^{u}-\widehat{T}_{A_{i}}^{l}\right)^{2}-\frac{1}{2}\left(1-\frac{1}{2} \widehat{T}_{A_{i}}^{u}-\frac{1}{2} \widehat{T}_{A_{i}}^{l}\right)
\end{array}\right\} \\
&=\max \left\{\begin{array}{c}
\left(\sum_{s=j}^{p} S\left(\left[\widehat{T}_{i s}^{l}, \widehat{T}_{i s}^{u}\right]\right)^{2} * \omega_{s}\right)^{2}-\frac{1-\sum_{r=j}^{p} H\left(\left[\widehat{T}_{i r}^{l}, \widehat{T}_{i r}^{u}\right]\right) * \omega_{r}}{2}, \\
4\left(\widehat{T}_{A_{i}}^{l}\right)^{2}+4\left(\widehat{T}_{A_{i}}^{u}\right)^{2}-8 \widehat{T}_{A_{i}}^{l} \widehat{T}_{A_{i}}^{u}-\frac{1}{2}-\frac{1}{4} \widehat{T}_{A_{i}}^{u}-\frac{1}{4} \widehat{T}_{A_{i}}^{l}
\end{array}\right\}
\end{aligned}
$$

where $0 \leq i \leq m$. The larger the value of $R\left(A_{i}\right)$, the more the suitability to which the alternative $A_{i}$ satisfies the decision-maker's requirement.

In equation (3), we know that the value of $R\left(A_{i}\right)$ depends on the value of $\left(\left(W\left(A_{i}\right)\right)^{2}-\frac{1-T\left(A_{i}\right)}{2}\right)$. So, next, we will point out how to obtain the optimal weights $\omega_{j}, \omega_{k}, \ldots, \omega_{p}$ for criteria $C_{j}, C_{k}, \ldots, C_{p}$ so that we can obtain the maximum value of above formula.

The optimal weights value can be computed via the following programming:

$$
\max \sum_{i=1}^{m}\left(\begin{array}{c}
\left(4\left(T_{A_{i j}}^{u}-T_{A_{i j}}^{l}\right)^{2}-\frac{1}{2}\left(1-\frac{1}{2} T_{A_{i j}}^{u}-\frac{1}{2} T_{A_{i j}}^{l}\right)\right) * \omega_{j} \\
+\left(4\left(T_{A_{i k}}^{u}-T_{A_{i k}}^{l}\right)^{2}-\frac{1}{2}\left(1-\frac{1}{2} T_{A_{i k}}^{u}-\frac{1}{2} T_{A_{i k}}^{l}\right)\right) * \omega_{k}+\ldots \\
+\left(4\left(T_{A_{i p}}^{u}-T_{A_{i p}}^{l}\right)^{2}-\frac{1}{2}\left(1-\frac{1}{2} T_{A_{i p}}^{u}-\frac{1}{2} T_{A_{i p}}^{l}\right)\right) * \omega_{p}
\end{array}\right),
$$

subject to the conditions

$$
\begin{gathered}
\omega_{j}^{l} \leq \omega_{j} \leq \omega_{j}^{u}, \\
\omega_{k}^{l} \leq \omega_{k} \leq \omega_{k}^{u}, \\
\vdots \\
\omega_{p}^{l} \leq \omega_{p} \leq \omega_{p}^{u},
\end{gathered}
$$

We can easily solve the above Linear Programming for example by using simplex method. 
The algorithm describing neut-MCDM is given as follows:

Name : neut-MCDM

Input : Netrosophic set of alternatives, Neutrosophic set of criteria

Output : Ordered list of alternatives, most preferred as the first element

1. $\left\{a_{\left(T_{i}, I_{i}, F_{i}\right)}^{i}: i=1, \cdots, m\right\}$ be nutrosophic set of alternatives with evaluations as neutrosophic components.

2. $\quad\left\{c_{\left(\widehat{T}_{j}, \widehat{I}_{j}, \widehat{F}_{j}\right)}: j=1, \cdots, n\right\}$ be nutrosophic set of criteria and their evaluations.

3. $\left[T_{i j}^{l}, T_{i j}^{u}\right]=\left[\min \left(\left(\frac{T_{A_{i j}}+I_{A_{i j}}}{2}\right),\left(\frac{1-F_{A_{i j}}+I_{A_{i j}}}{2}\right)\right), \max \left(\left(\frac{T_{A_{i j}}+I_{A_{i j}}}{2}\right),\left(\frac{1-F_{A_{i j}}+I_{A_{i j}}}{2}\right)\right)\right]$

4. $\quad$ For $i$ from 1 to $m$ do

5. $\quad$ For $j$ from 1 to $n$ do

6. Use scoring function: $S\left(A^{i j}\right)=2\left(T_{i j}^{u}-T_{i j}^{l}\right)$ to compute matrix $\mathbb{S}$ of scores.

7. $\quad$ Use accuracy function: $H\left(A^{i j}\right)=\frac{1}{2}\left(T_{i j}^{l}+T_{i j}^{u}\right)$ to compute matrix $\mathbb{H}$ of accuracies.

8. $\quad$ Using $\mathbb{S}, \mathbb{H}$ and $W\left(A^{i j}\right)=\left(S\left(A^{i j}\right)\right)^{2}-\frac{1-H\left(A^{i j}\right)}{2}$ compute and save matrix $\mathbb{W}$.

9. $\quad$ End For $j$

10. $\quad$ End For $i$

11. For $j$ from 1 to $n$ do

12. Compute and save criteria weights

13. $\quad\left[w_{j}^{l}, w_{j}^{u}\right]=\left[\min \left(\left(\frac{\widehat{T}_{j}+\widehat{I}_{j}}{2}\right),\left(\frac{1-\widehat{F}_{j}+\widehat{I}_{j}}{2}\right)\right), \max \left(\left(\frac{\widehat{T}_{j}+\widehat{I}_{j}}{2}\right),\left(\frac{1-\widehat{F}_{j}+\widehat{I}_{j}}{2}\right)\right)\right]$

14. End For $j$

15. Solve the following Linear Programming and get $w_{*}^{j}$

16. $\quad \max \sum_{j=1}^{n}\left(\sum_{i=1}^{n} w\left(A^{i j}\right)\right) w^{j}$

17. subject to

18. $\quad w_{j}^{l} \leq w^{j} \leq w_{j}^{u}$

19. Calculate $R\left(A^{i}\right)=\sum_{j=1}^{m}\left(\left(2\left(T_{i j}^{u}-T_{i j}^{l}\right)\right)^{2}-\frac{1-\frac{\left(T_{i j}^{l}+T_{i j}^{u}\right)}{2}}{2}\right) w_{*}^{j}$

20. Order $A^{i}$ with respect to $R\left(A^{i}\right)$. 


\section{Application}

In this section we present an illustrative example to explain the practical application of neut-MCDM.

Consider the evaluation of university professors for tenure and promotions. The criteria used at some universities are teaching, research, service and social participation. Weights must be determined for these criteria, and the candidates must also be evaluated with regard to each criterion. This example involves four criteria viz. Teaching, Research, Service, Social Participation and and three faculty candidates $A_{1}, A_{2}, A_{3}$. Let the Decision Matrix be given as

$$
\left.\begin{array}{ccccc}
\multicolumn{1}{c}{C_{1}} & C_{2} & C_{3} & C_{4} \\
& A_{1} \\
A_{3} & {[0.75,0.39,0.1]} & {[0.6,0.5,0.25]} & {[0.8,0.4,0.2]} & {[0.4,0.6,0.3]} \\
{[0.8,0.6,0.15]} & {[0.68,0.46,0.2]} & {[0.45,0.1,0.5]} & {[0.5,0.4,0.8]} \\
{[0.4,0.8,0.45]} & {[0.75,0.9,0.05]} & {[1,0.5,1]} & {[0.5,0.6,0.9]}
\end{array}\right]
$$

and the neutrosophic set describing the "importance of criteria" is given as

$$
\left\{\frac{C_{1}}{0.25,0.3,0.25}, \frac{C_{2}}{0.35,0.6,0.41}, \frac{C_{3}}{0.32,0.55,0.67}, \frac{C_{4}}{0.64,0.98,0.57}\right\}
$$

\begin{tabular}{|c|c|c|c|c|}
\hline & $C_{1}$ & $C_{2}$ & $C_{3}$ & $C_{4}$ \\
\hline$T$ & 0.25 & 0.35 & 0.32 & 0.64 \\
\hline$I$ & 0.30 & 0.60 & 0.55 & 0.98 \\
\hline$F$ & 0.25 & 0.41 & 0.67 & 0.57 \\
\hline
\end{tabular}

For simplifying computation, the neutrosophic set may be written as

Evaluation of each alternative i.e. $E\left(A_{i j}\right)=\left[T_{A_{i j}}^{l}, T_{A_{i j}}^{u}\right]$ is calculated first as:

$$
\left[T_{\left.A_{i j}, T_{A_{i j}}^{u}\right]}^{l}=\left[\begin{array}{cccc}
{[0.57,0.645]} & {[0.55,0.625]} & {[0.6,0.6]} & {[0.5,0.65]} \\
{[0.7,0.725]} & {[0.57,0.63]} & {[0.275,0.3]} & {[0.3,0.6]} \\
{[0.6,0.675]} & {[0.825,0.925]} & {[0.25,0.75]} & {[0.35,0.75]}
\end{array}\right]\right.
$$

Using the evaluations score and accuracy matrices $\mathbb{S}, \mathbb{H}$ are found

$$
\mathbb{S}=\left[\begin{array}{cccc}
0.15 & 0.15 & 0 & 0.3 \\
0.05 & 0.12 & 0.05 & 0.6 \\
0.15 & 0.2 & 1 & 0.8
\end{array}\right], \mathbb{H}=\left[\begin{array}{cccc}
0.6075 & 0.5875 & 0.6 & 0.575 \\
0.7125 & 0.6 & 0.2875 & 0.45 \\
0.6375 & 0.875 & 0.5 & 0.55
\end{array}\right]
$$


These matrices, in turn, let us to find the weight matrix $\mathbb{W}$

$$
\mathbb{W}=\left[\begin{array}{cccc}
-0.174 & -0.184 & -0.200 & -0.123 \\
-0.141 & -0.186 & -0.354 & 0.085 \\
-0.159 & -0.022 & 0.750 & 0.415
\end{array}\right]
$$

From $\mathbb{W}$ we get the coefficients of the linear programming problem as

$$
\begin{array}{llll}
-0.474 & -0.392 & 0.196 & 0.378
\end{array}
$$

The weights vary within following intervals:

$$
\begin{aligned}
& w_{1} \in[0.275,0.525] \\
& w_{2} \in[0.475,0.595] \\
& w_{3} \in[0.435,0.44] \\
& w_{4} \in[0.705,0.81]
\end{aligned}
$$

Thus the linear programming now can be set as:

$$
\begin{array}{cc}
\text { Maximize } & -0.474 w_{1}-0.392 w_{2}+0.196 w_{3}+0.378 w_{4} \\
\text { subject to } & 0.275 \leq w_{1} \leq 0.525 \\
0.475 & \leq w_{2} \leq 0.595 \\
& 0.435 \leq w_{3} \leq 0.44 \\
0.705 & \leq w_{4} \leq 0.81
\end{array}
$$

Maximum is at: $w_{1}=0.275, w_{2}=0.475, w_{3}=0.44, w_{4}=0.81$. Then for ranking the alternatives against the given criterion we have

$$
\begin{aligned}
& R\left(A_{1}\right)=\left((0.15)^{2}-\left(\frac{1-0.6075}{2}\right)\right) \times 0.275+\left((0.15)^{2}-\frac{1-0.5875}{2}\right) \times 0.475 \\
&+\left((0)^{2}-\frac{1-0.6}{2}\right) \times 0.44+\left((0.3)^{2}-\frac{1-0.575}{2}\right) \times 0.81=-0.3222875 \\
& R\left(A_{2}\right)=\left((0.05)^{2}-\left(\frac{1-0.7125}{2}\right)\right) \times 0.275+\left((0.12)^{2}-\left(\frac{1-0.6}{2}\right)\right) \times 0.475 \\
&+\left((0.05)^{2}-\left(\frac{1-0.2875}{2}\right)\right) \times 0.44+\left((0.6)^{2}-\left(\frac{1-0.45}{2}\right)\right) \times 0.81=-0.21380375 \\
& R\left(A_{3}\right)=\left((0.15)^{2}-\left(\frac{1-0.6375}{2}\right)\right) \times 0.275+\left((0.2)^{2}-\left(\frac{1-0.875}{2}\right)\right) \times 0.475 \\
&+\left((1)^{2}-\left(\frac{1-0.5}{2}\right)\right) \times 0.44+\left((0.8)^{2}-\left(\frac{1-0.55}{2}\right)\right) \times 0.81=0.61180625
\end{aligned}
$$


Therefore we can see that the alternative $A_{3}$ is the best choice. And the optimal ranking order of the alternatives is given by $A_{3} \succ A_{2} \succ A_{1}$. From the process of calculation, we can see that the method presented in this paper is extends a flexibility to set the criterion weights i.e importance for the given problem.

Conclusion 8. Contribution of the paper is twofold: it first introduces the theory of Neutrosophic Sets to MCDM community in a coherent manner. This would pave way for the future researchers in this area of investigation. Secondly, this paper presents a MCDM method based upon neutrosophic sets. To the best of our knowledge, this is a ground breaking work in neutrosophic MCDM. In the method the characteristics of the alternatives are represented by neutrosophic sets. The proposed method allows the degree of satisfiability, non satisfiability and indeterminacy of each alternative with respect to a set of criteria to be represented by neutrosophic sets, respectively. It allows the decision maker to assign and adjust the degree of satisfiability, non satisfiability and indeterminacy of the criteria to a vague concept "importance of criteria". For computer implementation of the method its algorithm viz. neut-MCDM has also been given. Using this algorithm, an example is presented to illustrate the neutrosophic decision-making process. From these we can see that the method differs from previous approaches for multicriteria decision making not only because it uses neutrosophic set theory, but also due to the fact that degree of importance of the criteria are not constant and the calculation is flexible and simpler.

\section{REFERENCES}

[1] F. Smarandache, AUnifying Field in Logics: Neutrosophic Logic. Neutrosophy, Neutrosophic Set, Neutrosophic Probability, American Research Press, Rehoboth, NM, 1999 .

[2] K.T. Atanassov, C. Cornelis, E.E. Kerre, Intuitionistic fuzzy sets and interval-valued fuzzy sets: a critical comparison, in: M. Wagenknecht, R.Hampel (Eds.), Proc. Third Internat. Conf. on Fuzzy Logic and Technology, 2003, pp. 159-163.

[3] W.B. Kandasamy, F. Smarandache, Neutrosophic Algebraic Structures, Hexis, Phoenix, 2006.

[4] F. Smarandache, R. Sunderraman, H. Wang, Y. Zhang, Interval Neutrosophic Sets and Logic: Theory and Applications in Computing, HEXIS Neutrosophic Book Series, No. 5, Books on Demand, Ann Arbor, MI, 2005.

[5] M. Khoshnevisan, S. Bhattacharya, A short note on financial data set detection using neutrosophic probability, in: F. Smarandache (Ed.), Proceedings of the First International Conference on Neutrosophy, Neutrosophic Logic, Neutrosophic Set, Neutrosophic Probability and Statistics, University of New Mexico, 2002, pp. 75-80.

[6] M. Khoshnevisan, S. Singh, Neurofuzzy and neutrosophic approach to compute the rate of change in new economies, in: F. Smarandache (Ed.), Proceedings of the First International Conference on Neutrosophy, Neutrosophic Logic, Neutrosophic Set, Neutrosophic Probability and Statistics, University of New Mexico, 2002, pp. $56-62$.

[7] Y.Guo, H.D.Cheng, New neutrosophic approach to image segmentation, Pattern Recognition 42 (2009) 587-595. 
[8] Y. Guo, H.D. Cheng, A new neutrosophic approach to image denoising, New Mathematics and Natural Computation 5 (3) (2009) 653-662.

[9] H.D. Cheng, Y. Guo, A new neutrosophic approach to image thresholding, New Mathematics and Natural Computation 4 (3) (2008) 291-308.

[10] M.Zhang, L.Zhang, H.D.Cheng, A neutrosophic approach to image segmentation based on watershed method, Signal Processing 90 (2010) 1510-1517.

[11] Athar Kharal, An application of neutrosophic sets in medical diagnosis, (submitted).

[12] U.Rivieccio, Neutrosophic logics: Prospects and problems, Fuzzy Sets and Systems 159 (2008) 1860-1868.

[13] P.Kraipeerapun, C.C.Fung, Binary classification using ensemble neural networks and interval neutrosophic sets, Neurocomputing 72 (2009) 2845-2856.

[14] A. Sengur, Y. Guo, Color texture image segmentation based on neutrosophic set and wavelet transformation, Computer Vision and Image Understanding 115 (2011) 11341144 .

[15] K.T. Atanassov, Intuitionistic fuzzy sets, in: VII ITKR's Session, Sofia, June 1983 (Deposed in Central Sci. Techn. Library of Bulg. Acad. of Sci., 1697/84) (in Bulgarian).

[16] Florentin Smarandache, "A Unifying field in logics. Neutrosophy: neutrosophic probability, set, and logic", Multiple-Valued Logic: An International Journal, 8(3) (2002) 385-438.

[17] Florentin Smarandache, Neutrosophic Set - A Generalization of the Intuitionistic Fuzzy Set, Proceedings of the First International Conference on Neutrosophy, Neutrosophic Logic, Neutrosophic Set, Neutrosophic Probability and Statistics, University of New Mexico, 2002. 